\title{
The Pitting Susceptibility Investigation of Passive Films Formed on X70, X80, and X100 Pipeline Steels by Electrochemical Noise and Mott-Schottky Measurements
}

\author{
Yang Zhao, ${ }^{1}$ Ping Liang, ${ }^{1}$ Yanhua Shi, ${ }^{1}$ Yunxia Zhang, ${ }^{1}$ and Tao Yang ${ }^{2}$ \\ ${ }^{1}$ School of Mechanical Engineering, Liaoning Shihua University, Fushun 113001, China \\ ${ }^{2}$ Liaoning Academy of Analytical Science, Shenyang 110016, China \\ Correspondence should be addressed to Ping Liang; liangping770101@163.com
}

Received 26 March 2015; Revised 14 June 2015; Accepted 18 June 2015

Academic Editor: Flavio Deflorian

Copyright (c) 2015 Yang Zhao et al. This is an open access article distributed under the Creative Commons Attribution License, which permits unrestricted use, distribution, and reproduction in any medium, provided the original work is properly cited.

The pitting susceptibility of passive films formed on X70, X80, and X100 pipeline steels was investigated by means of electrochemical noise (EN) and Mott-Schottky measurements. The EN results were analyzed according to the shot-noise theory and stochastic theory. Pit initiation process was analyzed quantitatively using the Weibull distribution function. Pit growth process was simulated by Gumbel distribution function. The experimental results of Mott-Schottky plots showed that the passive films formed on the three pipeline steels displayed an n-type semiconductor character, and the passive film for X100 pipeline steel has the lowest donor density $\left(N_{D}\right)$ among the three passive films. The EN results demonstrated that X100 pipeline steel had the lowest pit initiation rate and pit growth probability, which implied that the X100 pipeline steel had the lowest pitting susceptibility.

\section{Introduction}

In the recent years, extensive attentions have been paid to supply oil and gas in a more economic and safer way because of the continuously growing demand in energy consumption. Pipeline is an important way in oil and gas transportation over long distances [1]. In order to transport a greater volume of resources through pipelines, higher operating pressures and larger diameter of pipeline steel are required. Simultaneously, there is always demand from the industry to decrease pipeline construction and operating costs [2]. To date, X70 and X80 pipeline steels have been widely used in the project of oil/gas pipeline construction. X100 pipeline steel, which has a higher strength than X70 and X80 steel, without reducing toughness properties and weldability $[1,3,4]$, may have a good outlook for commercial application in the worldwide pipeline construction.

The construction of high strength steel pipes has been increasing in China, thus arousing unprecedented concern on pipeline security against corrosion damage in soil environments, which may affect the service safety of pipeline steels.
According to international investigations, stress corrosion cracking (SCC) is one of the most destructive deteriorations for oil and gas pipelines [5]. High $\mathrm{pH}$ SCC $(\mathrm{pH}$ greater than 9.0) has been recognized to be a typical failure mode, which was believed to be due to the selective dissolution and repeated rupture of passive film at the crack tips mechanism $[6,7]$. Many researchers $[8,9]$ considered that the resistance of SCC relied on the properties of passive films formed in concentrated carbonate/bicarbonate solutions. In the presence of aggressive species in the solutions, that is, $\mathrm{Cl}^{-}$ ions, the passive film may be broken and usually led to the occurrence of pitting $[9,10]$, which was probably the main cause of initiation of SCC [11]. Therefore, besides SCC, passive films properties and pitting behavior of pipeline steels have been the subjects of previous studies [2, 12-14]. For example, Zeng and Luo [12] demonstrated that the passive film formed on X70 pipeline steel in $0.5 \mathrm{~mol} / \mathrm{L} \mathrm{NaHCO}_{3}$ solution (a high $\mathrm{pH}$ value simulated soil solution) behaved like an $\mathrm{n}$-type semiconductor and had an electronic band structure. Xue and Cheng [13] confirmed that a stable passivity can be established on X80 pipeline steel in carbonate-bicarbonate 
TABLE 1: The chemical compositions of X70, X80, and X100 pipeline steels (wt. \%).

\begin{tabular}{lcccccccccccrr}
\hline Element & $\mathrm{C}$ & $\mathrm{Si}$ & $\mathrm{Mn}$ & $\mathrm{S}$ & $\mathrm{P}$ & $\mathrm{Ni}$ & $\mathrm{Cr}$ & $\mathrm{Cu}$ & $\mathrm{Nb}$ & $\mathrm{Al}$ & $\mathrm{Ti}$ & $\mathrm{Mo}$ & $\mathrm{Fe}$ \\
\hline X70 & 0.045 & 0.26 & 1.48 & 0.001 & 0.0017 & 0.16 & 0.17 & 0.21 & 0.033 & - & - & - & $\mathrm{Bal}$ \\
$\mathrm{X} 80$ & 0.06 & 0.08 & 1.87 & 0.005 & 0.006 & 0.16 & 0.03 & 0.26 & 0.044 & 0.03 & 0.01 & - & $\mathrm{Bal}$ \\
$\mathrm{X} 100$ & 0.05 & 0.25 & 1.83 & 0.002 & 0.08 & 0.23 & - & 0.21 & 0.10 & 0.02 & 0.018 & 0.27 & Bal. \\
\hline
\end{tabular}

solution, and the addition of chloride ions in the solution resulted in an increasing donor density in the passive film, which was prone to occurrence of pitting corrosion.

It is well known that pitting corrosion contains pit initiation and pit growth process [15]. The cycle polarization method is a common method for studying the pitting corrosion [16]; however, it does not provide much information about the occurrence of pitting and the rate of pit growth. Therefore, it is inadequate to use the method for studying pitting. More recently, electrochemical noise measurement (EN) technique, that is, the spontaneous fluctuations of the potential and/or the current generated during the uniform corrosion and pitting corrosion process, has the advantage of nonintrusive nature, quickness in gathering data, and ease interpretation, and it has been used in the studies of many research groups [17]. For example, Al-Mazeedi and Cottis [18] suggested that the EN measurement provides useful information about the rate of corrosion and localization of corrosion. Zhang et al. [15] used the EN to investigate the pitting susceptibility of Mg-10Gd-2Y-0.5Zr, AZ91D alloy, and pure magnesium in $0.05 \mathrm{M} \mathrm{NaCl}$ solution. Qafsaoui et al. [19] analyzed the effect of inhibitor on localized corrosion of $\mathrm{Al} 2024$ by EN, and Klapper et al. [20] considered the influence of the cathodic process on the interpretation of electrochemical noise signals arising from pitting corrosion of stainless steel.

Although some research related to the passive films and pitting of X70 and X80 pipeline steel has been performed [10-13], little attention has been paid to analyze the pitting corrosion of X100 pipeline steel in high $\mathrm{pH}$ soil environment $[21,22]$. Moreover, the EN method has not been widely used on the study of pitting for high strength pipeline steel. In the present work, the corrosion resistance for pitting of the passive film formed on X100 pipeline steels was investigated by means of electrochemical noise analysis combining with conventional electrochemical techniques with comparison of its counterpart X70 and X80 pipeline steel; the EN data were analyzed according to the stochastic theory and shot-noise theory, and the pitting corrosion was distinguished from uniform corrosion, and the pit initiation and pit growth process were estimated using Weibull and Gumbel distribution function, respectively. Therefore, the pitting susceptibility of three passive films was compared based on the EN data.

\section{Experimental}

2.1. Sample Pretreatment. X70, X80, and X100 pipeline steels were chosen as experimental specimens. The chemical compositions of the above materials are given in Table 1 . The three materials were embedded in two-component epoxy resin and mounted in a teflon (PTFE) holder, with an exposed area of $1.0 \mathrm{~cm}^{2}$. All the working surfaces were polished using fine grade emery papers of 240, 360, 600, 1000 and 1500 grit sizes. In order to ensure a clean pipeline steel surface before any exposure to the electrolyte solutions, the specimens were cleaned with ethanol after the polishing procedure.

The passive films were formed on X70, X80, and X100 pipeline steel in $0.5 \mathrm{~mol} / \mathrm{L} \mathrm{NaHCO}_{3}$ solution, which was used to simulate the composition of the high-pH soil. To make the pitting behavior of passive films be more evident, the electrochemical experiments including impedance measurement and $\mathrm{EN}$ measurements were carried out in $3.5 \mathrm{wt} . \% \mathrm{NaCl}$ solution. All of the solutions were prepared with analytical grade reagent and distilled water. The solutions were exposed to air and were quiescent in all the electrochemical experiments. All experiments were performed at room temperature.

2.2. Characterization. The microstructure of all the samples was observed with a Leica Q500MW metallurgical microscope. The electrochemical experiments were carried out in a conventional three-electrode system using EG\&G PARSTAT 2273 electrochemical workstation. The counter electrode was a graphite sheet and all the potentials are referred to the saturated calomel electrode (SCE). The working electrode was the three pipeline steel samples. Prior to all experiments, the working electrodes were prepolarized at $-1.3 \mathrm{~V}$ for $180 \mathrm{~s}$ to remove the surface oxide films formed in air and then the passive films were formed potentiostatically on the three pipeline steels at $0.7 \mathrm{~V}$ for $1 \mathrm{~h}$. The electrochemical impedance spectroscopy measurements were employed using a frequency range of $100 \mathrm{kHz}$ down to $10 \mathrm{mHz}$ and an amplitude of $10 \mathrm{mV}$; the EIS data was fitted using the ZSimpWin3.20 software. For Mott-Schottky measurements, a $5 \mathrm{mV}$ sine wave modulated signal was employed with a constant frequency of $1 \mathrm{kHz}$ and a step rate of $20 \mathrm{mV}$. Electrochemical noise measurements were performed using EN mode. The electrochemical current noise was traced as the galvanic coupling current between the two identical passive pipeline steels kept at the same potentials. EN data were instantaneously recorded with time for $1 \mathrm{~h}$. Each set of EN records, containing 100 data points, was kept with a data-sampling interval of $0.25 \mathrm{~s}$. 100-time record were analyzed. The corrosion micrographs after EN measurements were observed using TESCAN VEGA3SBH scanning electronic microscope (SEM).

\section{Results and Discussion}

3.1. Microstructure. The microstructural features of $\mathrm{X} 70$, $\mathrm{X} 80$, and X100 pipeline steels were examined with optical microscope, as shown in Figure 1. It can be seen that X70 steel 


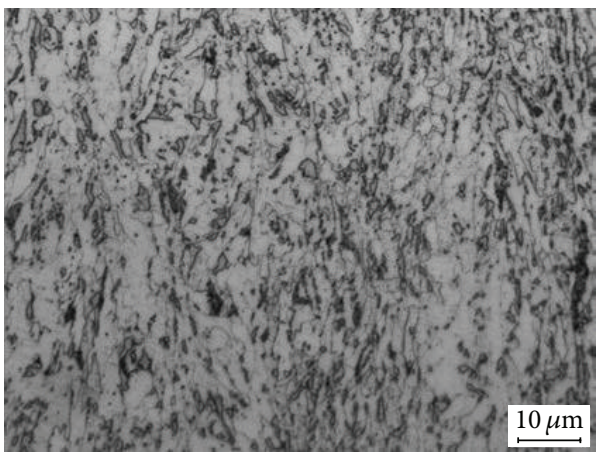

(a)

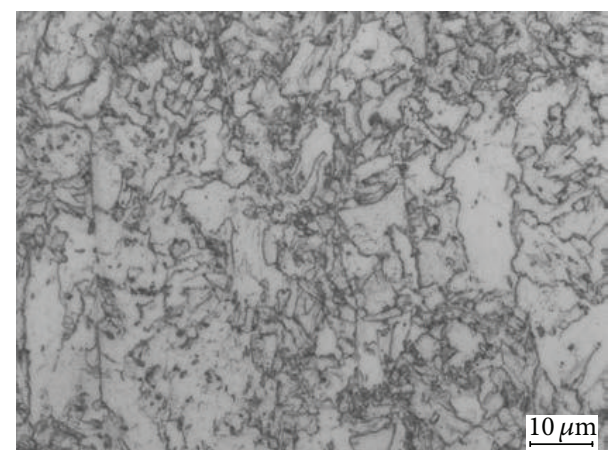

(b)

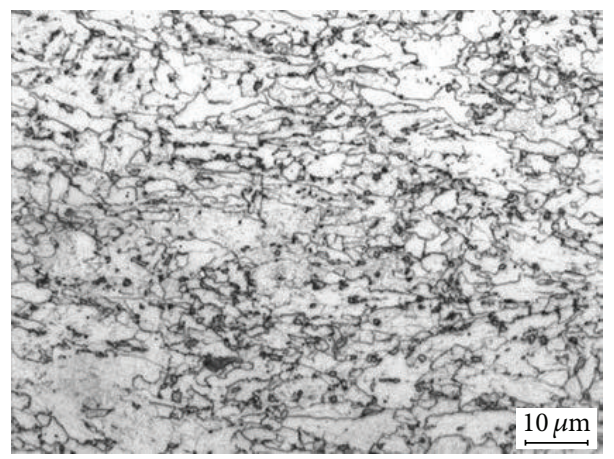

(c)

Figure 1: Optical micrograph of the microstructure for (a) X70, (b) X80, and (c) X100 pipeline steels.

(Figure 1(a)) exhibited a complex microstructure including fine acicular ferrite (AF) and granular bainite $(\mathrm{BF})$, which gave rise to high strength of pipeline steel $[23,24]$. The microstructure of X80 steel (Figure 1(b)) may be a combination of polygonal ferrite (PF) and granular bainite (BF), and X100 steel (Figure 1(c)) was also mainly composed of $\mathrm{AF}, \mathrm{BF}$, and martensitic-austenitic particles (M-A) distributed along the grain boundaries, and $\mathrm{M}-\mathrm{A}$ phases can improve significantly the strength of X100 pipeline steel as the second phase.

\subsection{Electrochemical Impedance Measurements of the Passive} Films. Electrochemical impedance spectroscopy (EIS) is an important technique to investigate the protective character of passive films against corrosion $[25,26]$. Figure 2 displays the Nyquist plots of the passive films in $3.5 \mathrm{wt} . \% \mathrm{NaCl}$ solution, and the passive oxide films were grown on X70, X80, and X100 pipeline steels at $+0.7 \mathrm{~V}$ in $0.5 \mathrm{~mol} / \mathrm{L} \mathrm{NaHCO}_{3}$ solution for $1 \mathrm{~h}$ to promote the passive film growth. It was noted that the shape of the plots for the three passive films was similar and all plots consisted of a single capacitive arc. The increasing diameter of the capacitive arc indicated that the corrosion resistance of passive films decreased in the following order: X100 > X80 > X70 pipeline steel.

The impedance spectra were fitted by the equivalent circuit diagram in Figure 3, where $R_{s}$ represents the solution resistance and $R_{f}$ and $Q_{f}$ represent the passive film resistance and the passive film capacitor, respectively. The impedance of

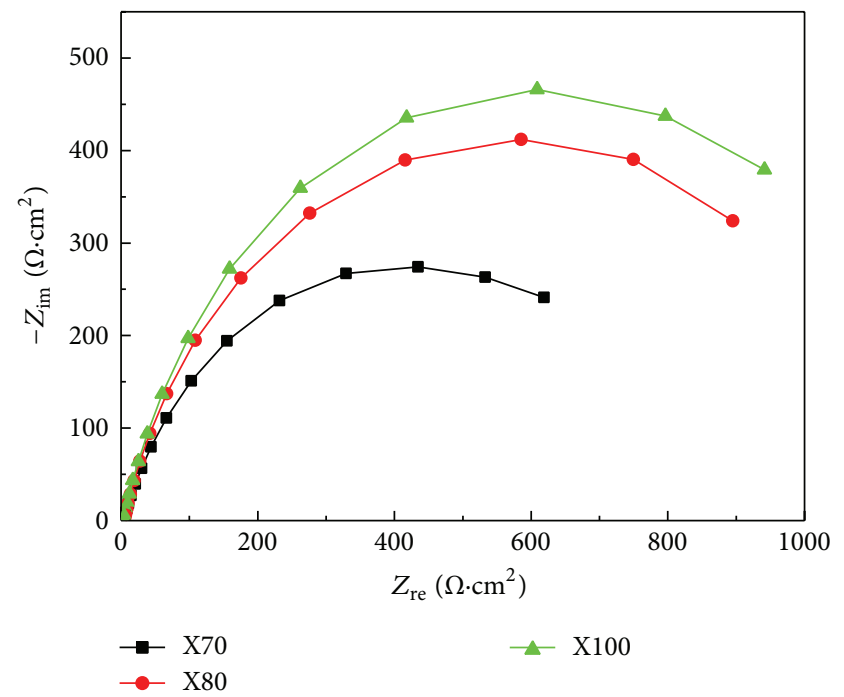

FIGURE 2: Nyquist plots of passive films for X70, X80, and X100 pipeline steels in $3.5 \mathrm{wt} . \% \mathrm{NaCl}$ solution.

the $Q_{f}$ is described mathematically as the following relation [27]:

$$
Q=\frac{(j \omega)^{-n}}{Y_{0}}
$$

$Y_{0}$ and $n$ are the constant and the value range of $n$ is $0<n<1$. 


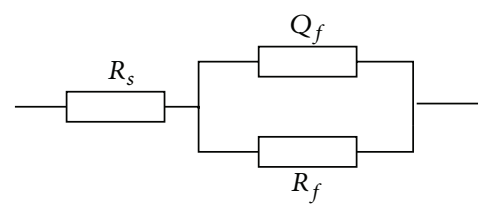

FIGURE 3: The equivalent circuit of electrochemical impedance spectroscopy.

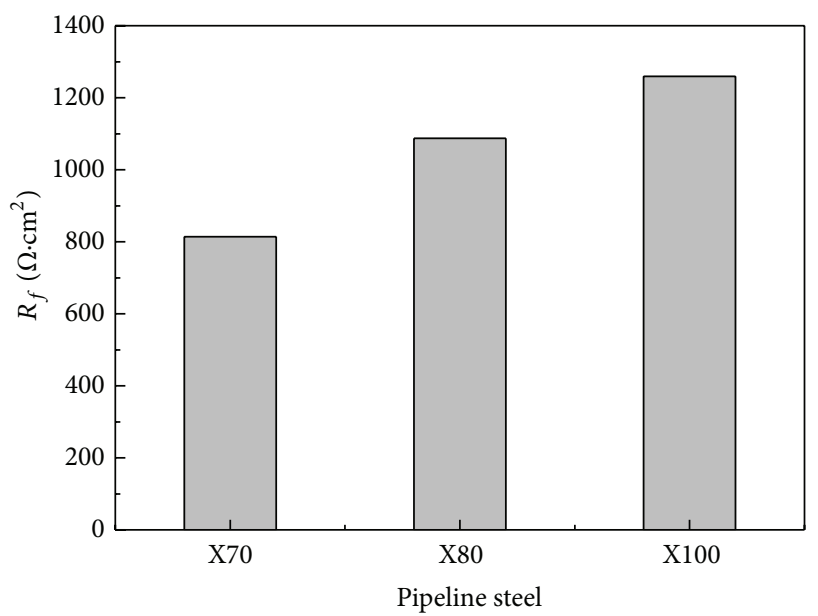

FIGURE 4: The resistance of passive films for X70, X80, and X100 pipeline steels in $3.5 \mathrm{wt} . \% \mathrm{NaCl}$ solution.

Figure 4 depicts the resistance $\left(R_{f}\right)$ of passive films for the three pipeline steels according to the fitted results of EIS. It was obvious that the resistance value of passive films for X100 steel was the highest, so it may be inferred that passive films of X100 pipeline steel were denser and more uniform than those of other two pipeline steels [28].

3.3. Semiconductor Properties of the Passive Films. The semiconductor behavior of the three passive films was investigated. Figure 5 provides the results of the capacitance measurements of the three passive films on X70, X80, and X100 pipeline steels. A linear behavior was observed from $0.2 \mathrm{~V}$ to $0.7 \mathrm{~V}$, and the positive slope of the linear segment indicated that the passive films expressed an n-type semiconductor character [29]. For an n-type semiconductor, the inverse of the square of the capacitance, $C^{-2}$, varied linearly with the applied electrode potential $(E)$, according to the equation derived from the Mott-Schottky model [30]. Assuming that the passive films were constituted by a defective $\gamma-\mathrm{Fe}_{2} \mathrm{O}_{3}$ or $\mathrm{Fe}_{3} \mathrm{O}_{4} / \gamma-\mathrm{Fe}_{2} \mathrm{O}_{3}$ that followed this model [12], the donor density $N_{D}$ of the passive films can be estimated from the slope of linearly fitted Mott-Schottky plots based on the following equation:

$$
\frac{1}{C^{2}}=\frac{2}{\varepsilon \varepsilon_{0} e N_{D}}\left(E-E_{\mathrm{fb}}-\frac{K T}{e}\right),
$$

where $\varepsilon$ is the dielectric constant of passive films, here $\varepsilon$ is taken as $15.6[12,29], \varepsilon_{0}$ is the vacuum permittivity constant $\left(8.85419 \times 10^{-12} \mathrm{~F} / \mathrm{m}\right), e$ is the elementary charge $(1.6 \times$
TABLE 2: The calculated donor density in the passive films for X70, $\mathrm{X} 80$, and X100 steels.

\begin{tabular}{lcc}
\hline Pipeline steels & Slope $\left(10^{9}\right)$ & $N_{D}\left(10^{21} N_{D} / \mathrm{cm}^{-3}\right)$ \\
\hline X70 & 6.667 & 1.357 \\
X80 & 8.889 & 1.018 \\
X100 & 14.444 & 0.626 \\
\hline
\end{tabular}

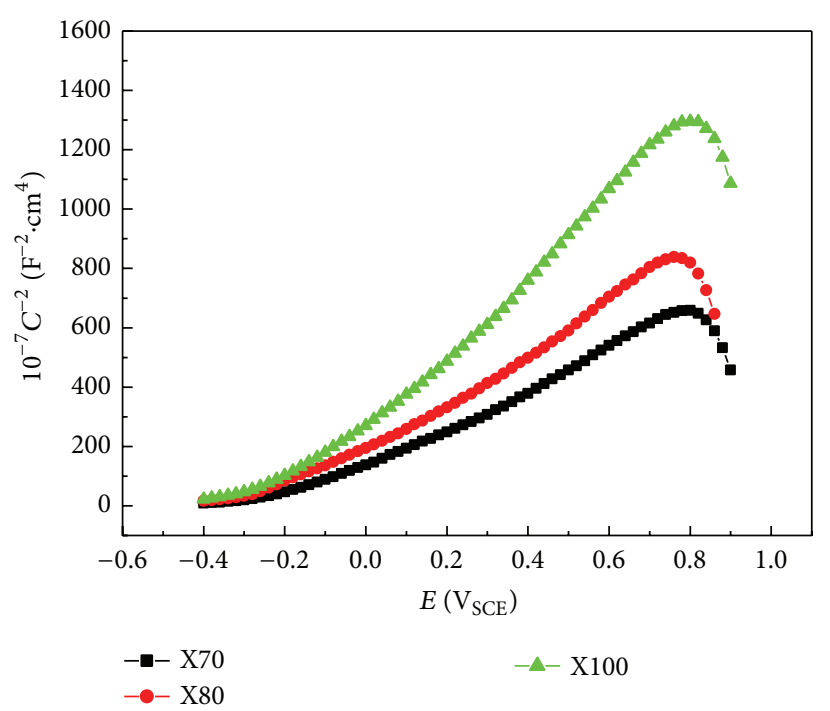

Figure 5: Mott-Schottky plots of the passive films formed on X70, $\mathrm{X} 80$, and X100 pipeline steels.

$10^{-19} \mathrm{C}$ ), $N_{D}$ is the donor density of donors, $E$ is the external electrode potential applied, $E_{\mathrm{fb}}$ is the flat band potential, $K_{B}$ is the Boltzmann's constant $\left(1.38 \times 10^{-23} \mathrm{~J} / \mathrm{K}\right), T$ is the absolute temperature $(298 \mathrm{~K})$, and $K T / q$ is about $25 \mathrm{mV}$ at room temperature, which can be negligible.

According to the PDM, $N_{D}$ corresponded to the point defects in the passive films [30], and the possible defect in passive film was oxygen vacancy and/or interstitial iron for n-type semiconductor. However, it was impossible for interstitial iron because the iron atoms were so large that there were few of them in interstitial positions due to the energy barrier. Thus, the primary defect in the passive films for pipeline steel is represented by oxygen vacancies [31, 32]. Generally, the defects were potential sites where film breakdown and pitting initiation were supposed to occur, so the smaller the $N_{D}$, the lower the chances of these processes. The calculated results of $N_{D}$ for the three passive films are shown in Table 2. It was clear that the $N_{D}$ was lower for passive films grown on X100 pipeline steel than X70 and X80 steel, meaning that the passive film of X100 steel had a decreasing concentration of oxygen vacancies and a more stable structure. Thence, it may be inferred that the passive film formed on X100 pipeline steel has a higher protection against pitting corrosion, compared with those passive films formed on X70 and X80 steel.

3.4. Electrochemical Noise Analysis on Corrosion Resistance of Passive Films. The electrochemical noise patterns of 


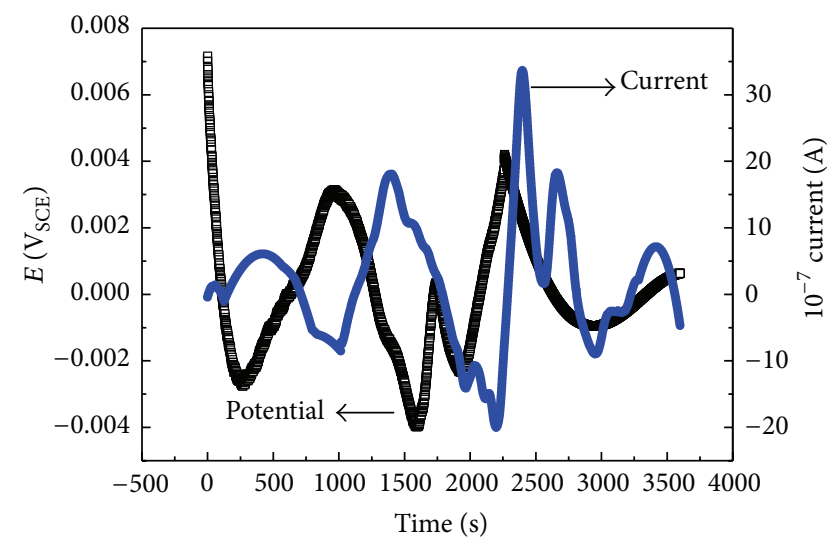

(a)

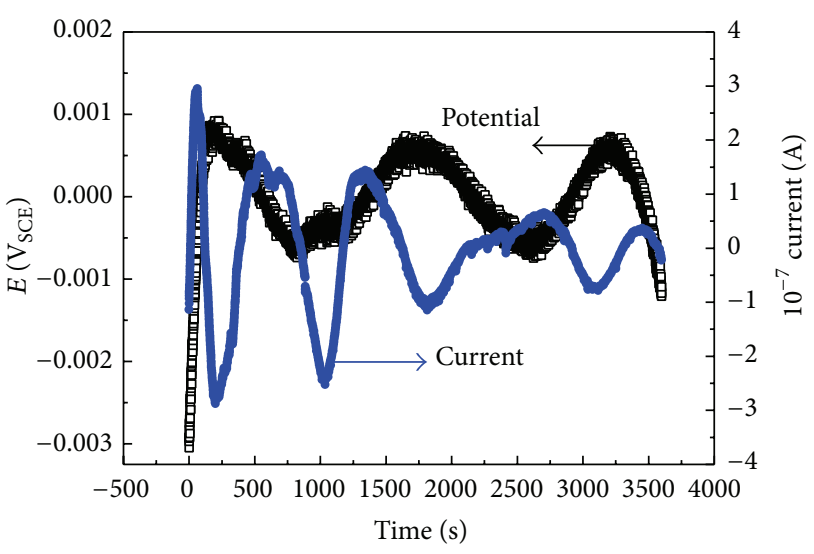

(b)

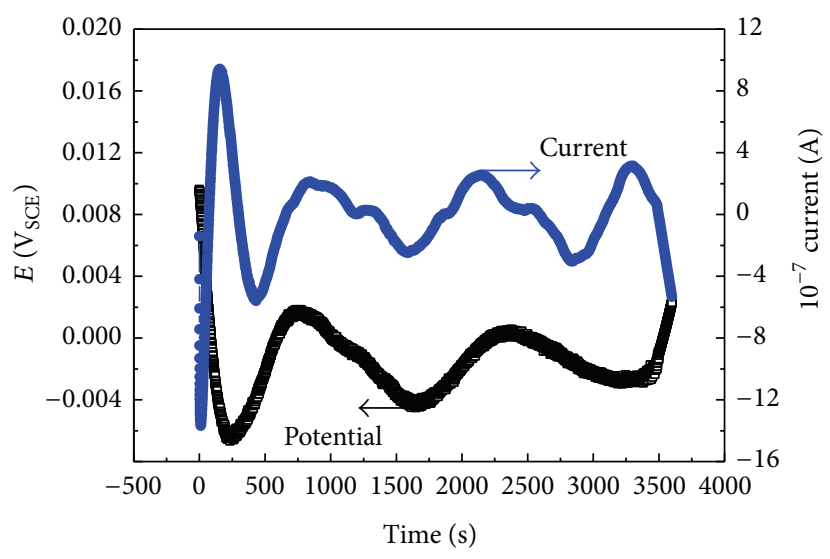

(c)

FIGURE 6: Electrochemical noise pattern of the passive films formed on (a) X70, (b) X80, and (c) X100 pipeline steels.

the three passive films are shown in Figure 6. It can be found that the curve of passive films of X100 pipeline steel exhibited larger amplitude fluctuations, meaning that a competitive process between pit initiation and passive film repassivation was undertaking on the metal surface. In contrast, X70 and X80 pipeline steels showed smaller amplitude fluctuations, indicating that the repassivated process of passive films was weak [15].

The noise resistance $R_{n}$ was an important parameter for the electrochemical noise data analysis, which was defined as the ratio of a standard deviation of the potential noise to that of the current noise [17], and the $R_{n}$ was the inverse of the corrosion rate [33]. Therefore, the corrosion behavior of the pipeline steels can be forecasted through $R_{n}$ [34]. Figure 7 shows the instantaneous values of the reciprocal of the noise resistance, $1 / R_{n}$, for the three passive films during $1 \mathrm{~h}$ immersion period in $3.5 \mathrm{wt} . \% \mathrm{NaCl}$ solution. The test results displayed that the values of $1 / R_{n}$ for the passive films of X100 pipeline steel were lower than other two passive films of X70 $\left(5.0441 \times 10^{-4} \Omega^{-1} \cdot \mathrm{cm}^{-2}\right)$ and X80 $(2.1657 \times$ $10^{-4} \Omega^{-1} \cdot \mathrm{cm}^{-2}$ ) steel, indicating the corrosion rate of X100 pipeline steel was lower than other two steels.

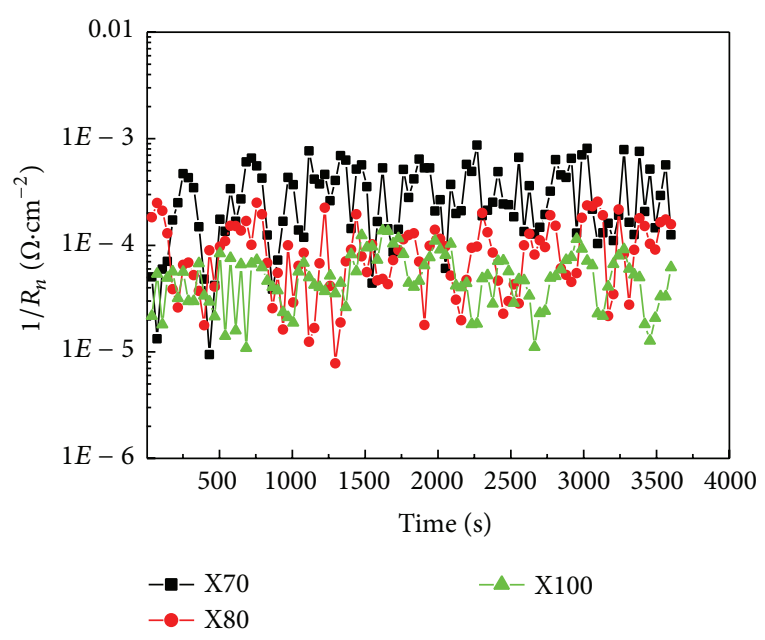

FIGURE 7: The reciprocal of noise resistance of passive films formed on (a) X70, (b) X80, and (c) X100 pipeline steels.

The another parameter $q$, that is, the corrosion charge (q) of corrosion events based on the shot-noise theory, gives 


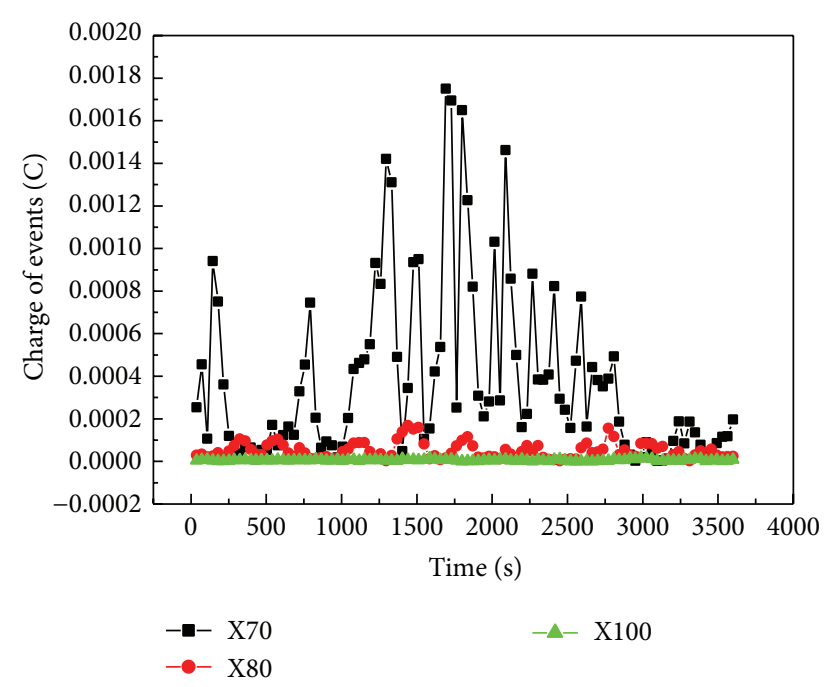

FIGURE 8: Corrosion charge ( $q$ ) of passive films formed on X70, X80, and X100 pipeline steels.

information of the mass of metal lost in the corrosion event using Faraday theory. It was calculated by the following equation:

$$
\begin{aligned}
\bar{I}_{\text {corr }} & =\bar{q} \times f_{n} \\
f_{n} & =\frac{\bar{I}_{\text {corr }}}{\bar{q}}=\frac{B^{2}}{\Psi_{E}} \\
q & =\frac{\sqrt{\varphi_{E}} \cdot \sqrt{\varphi_{I}}}{B},
\end{aligned}
$$

where $\bar{q}$ is the average charge in each event, $f_{n}$ is the frequency of events, and $\Psi_{E}$ and $\Psi_{I}$ are the low frequency limit $0.01 \mathrm{~Hz}$ of power spectral density of potential and current noise, respectively. $B$ is the Stern-Geary constant.

Figure 8 reveals the corrosion charge $(q)$ of the three passive films for X70, X80, and X100 pipeline steels. The results showed that the baseline (approximately $800 \mu \mathrm{C}$ ) for the corrosion charges of the passive film for $\mathrm{X} 70$ pipeline steel was about 10 times and 100 times larger than those for X80 and X100 steel, respectively, meaning that the X100 steel has a better corrosion resistance than X70 and X80 steel.

3.5. Stochastic Analysis for Pit Initiation Rate. Cottis and AlMazeedi $[18,35]$ considered that $f_{n}$ described the frequency of events. In general, it was expected that high frequency events would tend to occur all over the surface, and the corrosion would therefore be uniform. In contrast, low frequency events must be removing relatively large amounts of material at individual locations, and the corrosion would be rather localized. Thus, $f_{n}$ provided relatively good discrimination between uniform corrosion and local corrosion, and a small $f_{n}$ indicated localized corrosion, and a large $f_{n}$ meant uniform corrosion. The $f_{n}$ was plotted in Figure 9 as a function of the cumulative probability, $p$. It can be seen from Figure 9 that the values $f_{n}$ of passive films for X100 pipeline steel were slightly larger, and the range of values $f_{n}$ was also

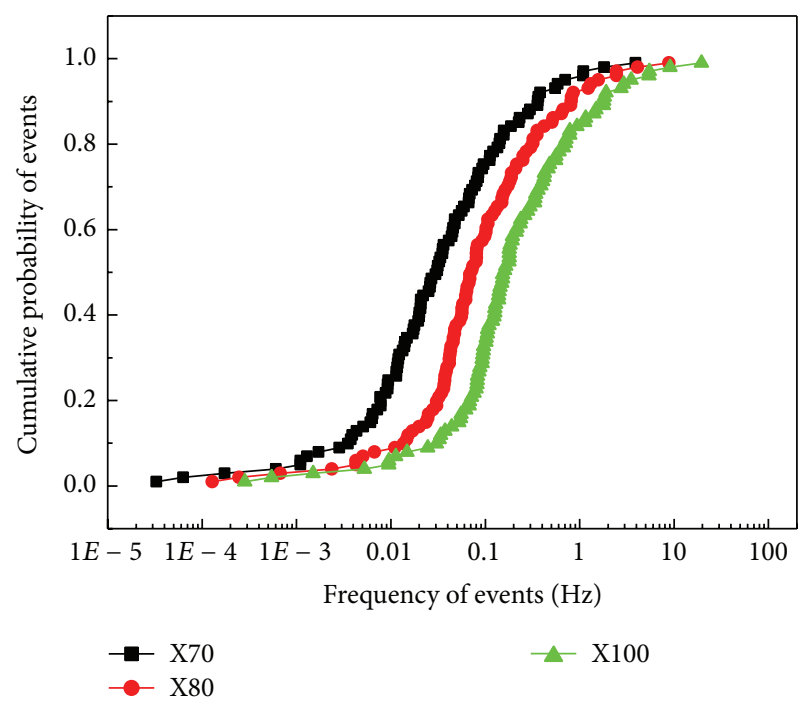

FIGURE 9: Cumulative probability plots of passive films formed on $\mathrm{X} 70, \mathrm{X} 80$, and X100 pipeline steels for the frequency of events, $f_{n}$.

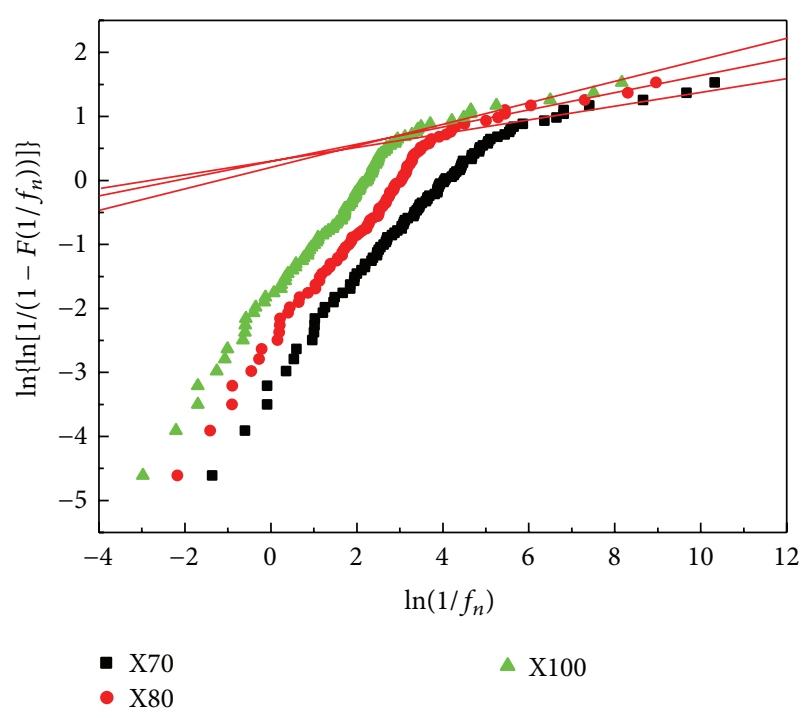

FIGURE 10: Weibull probability plots of X70, X80, and X100 pipeline steel passive film.

wider than those of other two pipeline steels, meaning that the X100 steel had further uniform corrosion, while X70 and X80 pipeline steels would tend to local corrosion, that is, pitting.

The stochastic analysis of nonhomogeneous Poisson process can be analyzed using Weibull distribution, which was considered to provide some information on pit initiation $[36,37]$. Figure 10 describes the Weibull probability plots $\ln \left\{\ln \left[1 /\left(1-F\left(1 / f_{n}\right)\right)\right]\right\}$ versus $\ln \left(1 / f_{n}\right)$ plots of the passive films for X70, X80, and X100 pipeline steels. It was found that all the plots showed two linear regions in one plot, which was likely to imply the existence of two failure modes, depending on $1 / f_{n}$. Because only uniform and pitting corrosion will occur during the noise measurement, these two slopes represented two limiting cases corresponding to dominant pitting 
TABLE 3: Weibull distribution parameters for pitting corrosion of passive films formed on X70, X80, and X100 pipeline steels.

\begin{tabular}{lcc}
\hline Materials & Shape parameter $m(-)$ & Shape parameter $n$ \\
\hline X70 & 0.0985 & 1.0558 \\
X80 & 0.1152 & 1.6241 \\
X100 & 0.1457 & 1.9997 \\
\hline
\end{tabular}

corrosion and dominant uniform corrosion, respectively [38]. It was suggested that the slopes in the relatively shorter $1 / f_{n}$ region were associated with uniform corrosion, and the slopes in the relatively longer $1 / f_{n}$ region were responsible for pitting corrosion such as metastable pitting or pit initiation [37]. It can be found that the line towards the left of the Figure 10 represented dominant uniform corrosion, and the line towards the right of the Figure 10 represented dominant pitting corrosion.

The conditional event generation rate $r(t)$ is employed as a kind of failure rate in reliability engineering, which is defined as

$$
r(t)=\frac{m}{n} t^{m-1}
$$

where $m$ and $n$ are the shape and scale parameters, respectively. $m$ is a dimensionless parameter and $n$ is expressed as $s_{m}$.

Based on the Weibull distribution function, the value of $r(t) d t$ represented the generation probability of the events in the next unit time $d t$ for the sample in which events have not been generated when $t$ has elapsed. This just corresponded to the pit embryo formation rate in pitting corrosion $[15,31,36]$.

The values of the shape parameter $m$ and scale parameter $n$ for pitting corrosion were estimated from Figure 10, which are listed in Table 3 . The pitting initiation rate $r(t)$ was determined by inserting the values of $m$ and $n$ given in Table 3 into (4), and the resulting $r(t)$ is illustrated as a function of time $t$ in Figure 11. It was observed that the value of $r(t)$ of passive films for X100 steel was obviously lowed compared to those of X70 and X80 pipeline steels at a given time $t$, which indicated that the metastable pitting or pit initiation was suppressed. Therefore, the pit initiation rate decreased in the following order: X70 > X80 > X100 steel.

3.6. Stochastic Analysis For Pit Growth. In order to describe the pit radius of the passive films formed on pipeline steels, the Gumbel extreme value distribution was used [39]. If the pits were assumed to be hemispherical, the pit radius can be calculated according to [15]

$$
\begin{aligned}
V\left(\mathrm{~cm}^{3}\right) & =\frac{q \times M}{F \times n \times \rho} \\
R_{\text {pit }}(\mu \mathrm{m}) & =\left(\sqrt[3]{\frac{3 \times V\left(\mathrm{~cm}^{3}\right)}{2 \pi}}\right) \times 10000,
\end{aligned}
$$

where $V$ is the volume of pit cavity, $q$ is the charge passed, $M$ is molecular mass, $F$ is Faraday constant, $\rho$ is density, and $R_{\text {pit }}$ is radius of pit cavity, respectively.

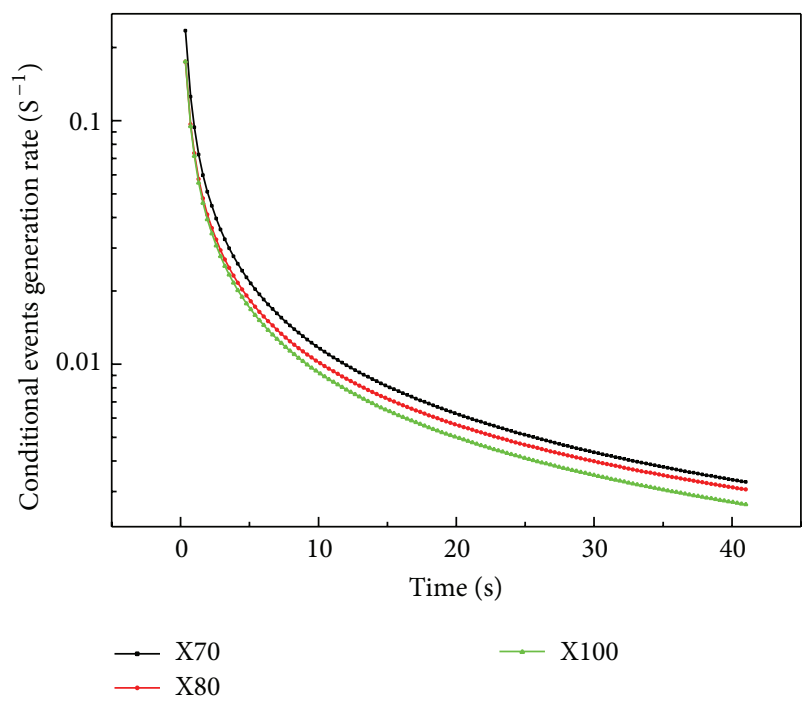

FIgURE 11: The plots of pit initiation rate of passive films for X70, $\mathrm{X} 80$, and X100 pipeline steel.

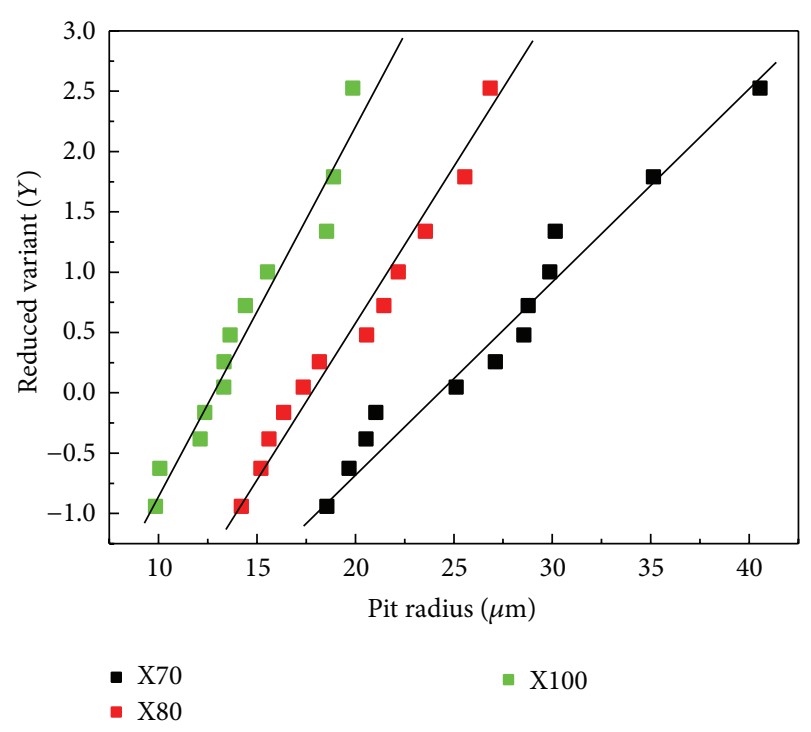

FIGURE 12: Gumbel probability plots of passive films for X70, X80, and X100 pipeline steel.

The largest pit sizes within each of the EN segments and the values were subjected to extreme value statistics analysis. The values of the reduced variant are plotted in Figure 12 against the ordered pit sizes. From Figure 12, it can be found that stable pit (radius $>10 \mathrm{um}$ ) occurred on the three passive films of X70, X80, and X100 pipeline steel, and the pit radius could be ranked in a reduced series: X70 > X80 > X100 steel.

3.7. Corrosion Morphology Observation. After the electrochemical noise measurements, the corrosion micrographs of the three passive films were observed by SEM (Figure 13). It was clear that the number of pits could be ranked in a reduced series: X70 > X80 > X100 steel. Moreover, X100 pipeline steel exhibited a smaller pit radius than that of X80 and X70 


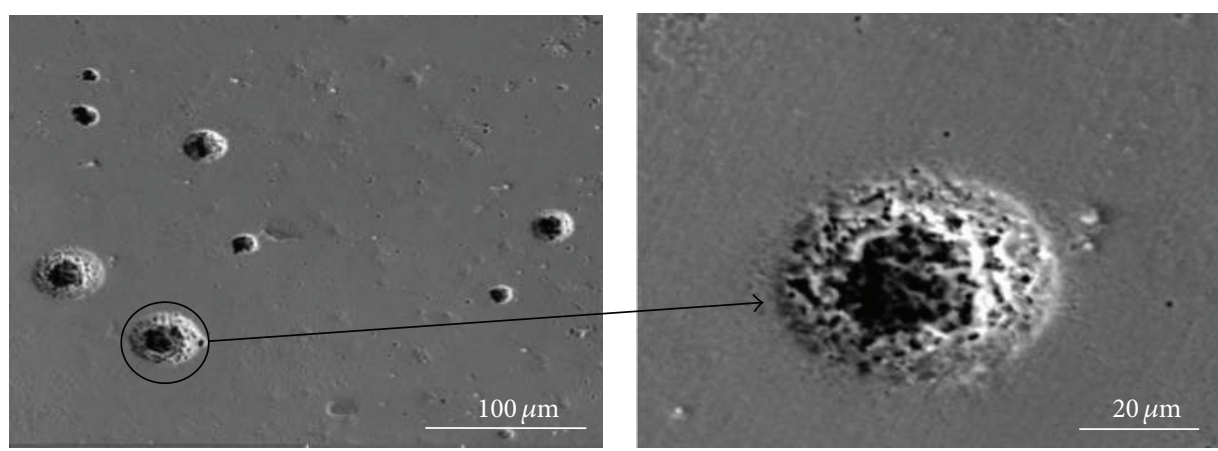

(a)

(b)

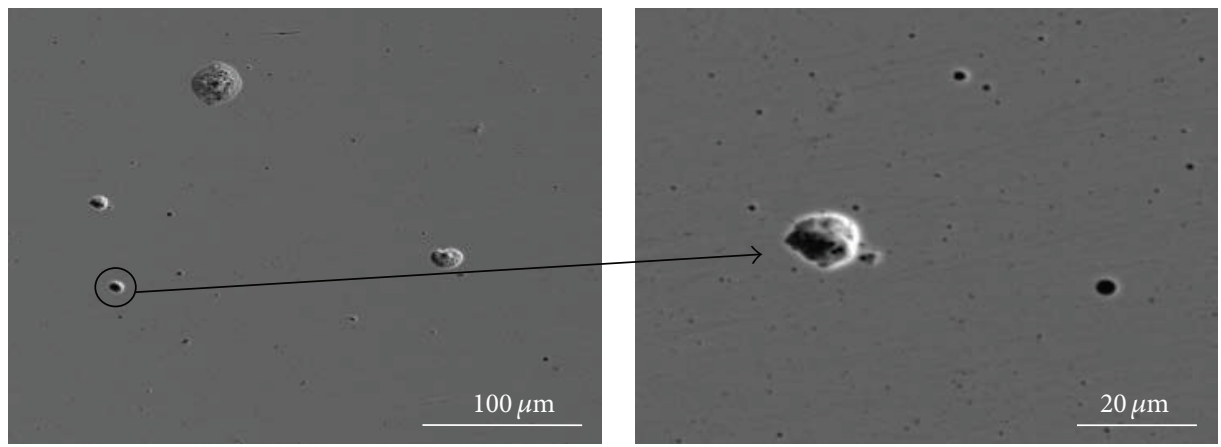

(c)

(d)

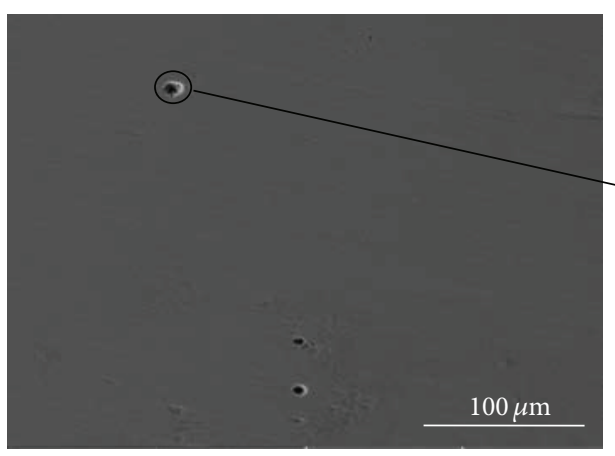

(e)

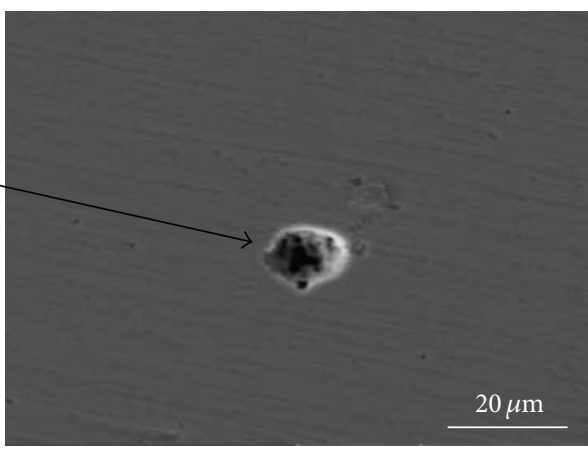

(f)

FIGURE 13: SEM images of corrosion morphology of passive films for X70, X80, and X100 pipeline steels after the electrochemical noise measurement for $1 \mathrm{~h}$ in $3.5 \mathrm{wt} . \% \mathrm{NaCl}$ solution (a) and (b) X70 steel; (c) and (d) X80 steel; (e) and (f) X100 steel.

steel. This meant that the stochastic analysis of EN results concerning pit initiation and pit growth was available.

Combining the results of EN data and SEM, the passive film for X100 pipeline steel displayed the lowest pit initiation rate and pit growth probability, which indicated that X100 pipeline steel had the lowest pit susceptibility followed by X80 and X70 pipeline steel.

\section{Conclusions}

The passive films formed on X70, X80, and X100 pipeline steel in $0.5 \mathrm{~mol} / \mathrm{L} \mathrm{NaHCO}_{3}$ solution expressed an n-type semiconductor character, and the passive film of X100 steel displayed a lower donor density than other two passive films for X70 and X80 pipeline steels.
The EN data obtained from three films have been analyzed based on the combined stochastic theory and shotnoise theory. The pitting process could be distinguished from the electrochemical noises according to the events of relatively lower frequency regions. The pit initiation process has been analyzed based on Weibull distribution function, while pit growth process has been analyzed using a Gumbel distribution function. These results implied that the pitting susceptibility of the passive films can be ranked as a decreasing series: X70 > X80 > X100 pipeline steel.

\section{Conflict of Interests}

The authors declare that there is no conflict of interests regarding the publication of this paper. 


\section{Acknowledgment}

The authors acknowledge the support of the General Science Study Project of Education Department of Liaoning Province (L2012127).

\section{References}

[1] C. Zhang and Y. F. Cheng, "Synergistic effects of hydrogen and stress on corrosion of X100 pipeline steel in a near-neutral ph solution," Journal of Materials Engineering and Performance, vol. 19, no. 9, pp. 1284-1289, 2010.

[2] F. Mohammadi, F. F. Eliyan, and A. Alfantazi, "Corrosion of simulated weld HAZ of API X-80 pipeline steel," Corrosion Science, vol. 63, no. 12, pp. 323-333, 2012.

[3] Z. Bi, H. Liu, X. Jing, and H. Niu, "Research on submerged arc welding wire for X100 pipeline steel," China Welding (English Edition), vol. 20, no. 2, pp. 56-62, 2011.

[4] T. Niu, Y.-L. Kang, H.-W. Gu, Y.-Q. Yin, and M.-L. Qiao, "Precipitation behavior and its strengthening effect of X100 pipeline steel," Journal of Iron and Steel Research International, vol. 17, no. 11, pp. 73-78, 2010.

[5] Z. Y. Liu, C. W. Du, X. Zhang, F. M. Wang, and X. G. Li, "Effect of $\mathrm{pH}$ value on stress corrosion cracking of X70 pipeline steel in acidic soil environment," Acta Metallurgica Sinica (English Letters), vol. 26, no. 4, pp. 489-496, 2013.

[6] B. T. Lu, "Further study on crack growth model of buried pipelines exposed to concentrated carbonate-bicarbonate solution," Engineering Fracture Mechanics, vol. 131, no. 12, pp. 296-314, 2014.

[7] C. W. Du, X. G. Li, P. Liang, Z. Y. Liu, G. F. Jia, and Y. F. Cheng, "Effects of microstructure on corrosion of X70 pipe steel in an alkaline soil," Journal of Materials Engineering and Performance, vol. 18, no. 2, pp. 216-220, 2009.

[8] G. A. Zhang and Y. F. Cheng, "Micro-electrochemical characterization and Mott-Schottky analysis of corrosion of welded X70 pipeline steel in carbonate/bicarbonate solution," Electrochimica Acta, vol. 55, no. 1, pp. 316-324, 2009.

[9] A. Q. Fu and Y. F. Cheng, "Electrochemical polarization behavior of X70 steel in thin carbonate/bicarbonate solution layers trapped under a disbonded coating and its implication on pipeline SCC," Corrosion Science, vol. 52, no. 7, pp. 2511-2518, 2010.

[10] P. Liang, X. G. Li, C. W. Du, X. Chen, and L. Zhang, "Influence of chloride ions on the corrosion resistance of X80 pipeline steel in $\mathrm{NaHCO}_{3}$ solution," Journal of University of Science and Technology, vol. 30, no. 7, pp. 735-739, 2008.

[11] A. Eslami, R. Kania, B. Worthingham, G. V. Boven, R. Eadie, and W. Chen, "Effect of $\mathrm{CO}_{2}$ and R-ratio on near-neutral $\mathrm{pH}$ stress corrosion cracking initiation under a disbonded coating of pipeline steel," Corrosion Science, vol. 53, no. 6, pp. 2318-2327, 2011.

[12] Y. M. Zeng and J. L. Luo, "Electronic band structure of passive film on X70 pipeline steel," Electrochimica Acta, vol. 48, no. 23, pp. 3551-3562, 2003.

[13] H. B. Xue and Y. F. Cheng, "Passivity and pitting corrosion of $\mathrm{X} 80$ pipeline steel in carbonate/bicarbonate solution studied by electrochemical measurements," Journal of Materials Engineering and Performance, vol. 19, no. 9, pp. 1311-1317, 2010.

[14] I. A. Chaves and R. E. Melchers, "Pitting corrosion in pipeline steel weld zones," Corrosion Science, vol. 53, no. 12, pp. 40264032, 2011.
[15] T. Zhang, X. L. Liu, Y. W. Shao, G. Z. Meng, and F. H. Wang, "Electrochemical noise analysis on the pit corrosion susceptibility of Mg-10Gd-2Y-0.5Zr, AZ91D alloy and pure magnesium using stochastic model," Corrosion Science, vol. 50, no. 12, pp. 3500-3507, 2008.

[16] F. F. Eliyan and A. Alfantazi, "Influence of temperature on the corrosion behavior of API-X100 pipeline steel in 1-bar $\mathrm{CO}_{2}$ $\mathrm{HCO}_{3}{ }^{-}$solutions: an electrochemical study," Materials Chemistry and Physics, vol. 140, no. 2-3, pp. 508-515, 2013.

[17] Y. W. Shao, C. Jia, G. Z. Meng, T. Zhang, and F. H. Wang, "The role of a zinc phosphate pigment in the corrosion of scratched epoxy-coated steel," Corrosion Science, vol. 51, no. 2, pp. 371-379, 2009.

[18] H. A. A. Al-Mazeedi and R. A. Cottis, "A practical evaluation of electrochemical noise parameters as indicators of corrosion type," Electrochimica Acta, vol. 49, no. 17-18, pp. 2787-2793, 2004.

[19] W. Qafsaoui, F. Huet, and H. Takenouti, "Analysis of the inhibitive effect of BTAH on localized corrosion of Al 2024 from electrochemical noise measurements," Journal of the Electrochemical Society, vol. 156, no. 2, pp. C67-C74, 2009.

[20] H. S. Klapper, J. Goellner, and A. Heyn, "The influence of the cathodic process on the interpretation of electrochemical noise signals arising from pitting corrosion of stainless steels," Corrosion Science, vol. 52, no. 4, pp. 1362-1372, 2010.

[21] F. F. Eliyan, E.-S. Mahdi, and A. Alfantazi, "Electrochemical evaluation of the corrosion behaviour of API-X100 pipeline steel in aerated bicarbonate solutions," Corrosion Science, vol. 58, no. 5, pp. 181-191, 2012.

[22] F. F. Eliyan and A. Alfantazi, "On the theory of $\mathrm{CO}_{2}$ corrosion reactions-investigating their interrelation with the corrosion products and API-X100 steel microstructure," Corrosion Science, vol. 85, pp. 380-393, 2014.

[23] K. Banerjee and U. K. Chatterjee, "Hydrogen permeation and hydrogen content under cathodic charging in HSLA 80 and HSLA 100 steels," Scripta Materialia, vol. 44, no. 2, pp. 213-216, 2001.

[24] M. Zhu, C. W. Du, X. G. Li et al., "Effect of strength and microstructure on stress corrosion cracking behavior and mechanism of X80 pipeline steel in high $\mathrm{pH}$ carbonate/bicarbonate solution," Journal of Materials Engineering and Performance, vol. 23, no. 4, pp. 1358-1365, 2014.

[25] S. Nagarajan, V. Raman, and N. Rajendran, "Evaluation of passive film behaviour of super austenitic stainless steels at different potential regions using dynamic electrochemical impedance spectroscopy," Journal of Solid State Electrochemistry, vol. 14, no. 7, pp. 1197-1204, 2010.

[26] Z.-J. Jia, C.-W. Du, C.-T. Li, Z. Yi, and X.-G. Li, "Study on pitting process of 316L stainless steel by means of staircase potential electrochemical impedance spectroscopy," International Journal of Minerals, Metallurgy and Materials, vol. 18, no. 1, pp. 48-52, 2011.

[27] C. N. Cao and J. Q. Zhang, An Introduction to Electrochemical Impedance Spectroscopy, Science Press, Beijing, China, 2004.

[28] L. Hamadou, A. Kadri, and N. Benbrahim, "Characterisation of passive films formed on low carbon steel in borate buffer solution ( $\mathrm{pH}$ 9.2) by electrochemical impedance spectroscopy," Applied Surface Science, vol. 252, no. 5, pp. 1510-1519, 2005.

[29] C. A. Gervasi, M. E. Folquer, A. E. Vallejo, and P. E. Alvarez, "Electron transfer across anodic films formed on tin in carbonate-bicarbonate buffer solution," Electrochimica Acta, vol. 50, no. 5, pp. 1113-1119, 2005. 
[30] D. D. Macdonald, "The point defect model for the passive state," Journal of the Electrochemical Society, vol. 139, no. 12, pp. 34343449, 1992.

[31] A. J. Davenport, L. J. Oblonsky, M. P. Ryan, and M. F. Toney, "Structure of the passive film that forms on iron in aqueous environments," Journal of the Electrochemical Society, vol. 147, no. 6, pp. 2162-2173, 2000.

[32] V. A. Alves and C. M. A. Brett, "Characterisation of passive films formed on mild steels in bicarbonate solution by EIS," Electrochimica Acta, vol. 47, no. 13-14, pp. 2081-2091, 2002.

[33] J. F. Chen and W. F. Bogaerts, "The physical meaning of noise resistance," Corrosion Science, vol. 37, no. 11, pp. 1839-1842, 1995.

[34] A.-M. Lafront, W. Zhang, S. Jin, R. Tremblay, D. Dubé, and E. Ghali, "Pitting corrosion of AZ91D and AJ62x magnesium alloys in alkaline chloride medium using electrochemical techniques," Electrochimica Acta, vol. 51, no. 3, pp. 489-501, 2005.

[35] R. A. Cottis, "Interpretation of electrochemical noise data," Corrosion, vol. 57, no. 3, pp. 265-285, 2001.

[36] J.-J. Park and S.-I. Pyun, "Stochastic approach to the pit growth kinetics of Inconel alloy 600 in $\mathrm{Cl}^{-}$ion-containing thiosulphate solution at temperatures $25-150 \circ \mathrm{C}$ by analysis of the potentiostatic current transients," Corrosion Science, vol. 46, no. 2, pp. 285-296, 2004.

[37] K.-H. Na, S.-I. Pyun, and H.-P. Kim, "Analysis of electrochemical noise obtained from pure aluminium in neutral chloride and alkaline solutions," Corrosion Science, vol. 49, no. 1, pp. 220-230, 2007.

[38] Y. W. Shao, H. Huang, T. Zhang, G. Z. Meng, and F. H. Wang, "Corrosion protection of $\mathrm{Mg}-5 \mathrm{Li}$ alloy with epoxy coatings containing polyaniline," Corrosion Science, vol. 51, no. 12, pp. 2906-2915, 2009.

[39] G. Engelhardt and D. D. Macdonald, "Unification of the deterministic and statistical approaches for predicting localized corrosion damage. I. Theoretical foundation," Corrosion Science, vol. 46, no. 11, pp. 2755-2780, 2004. 

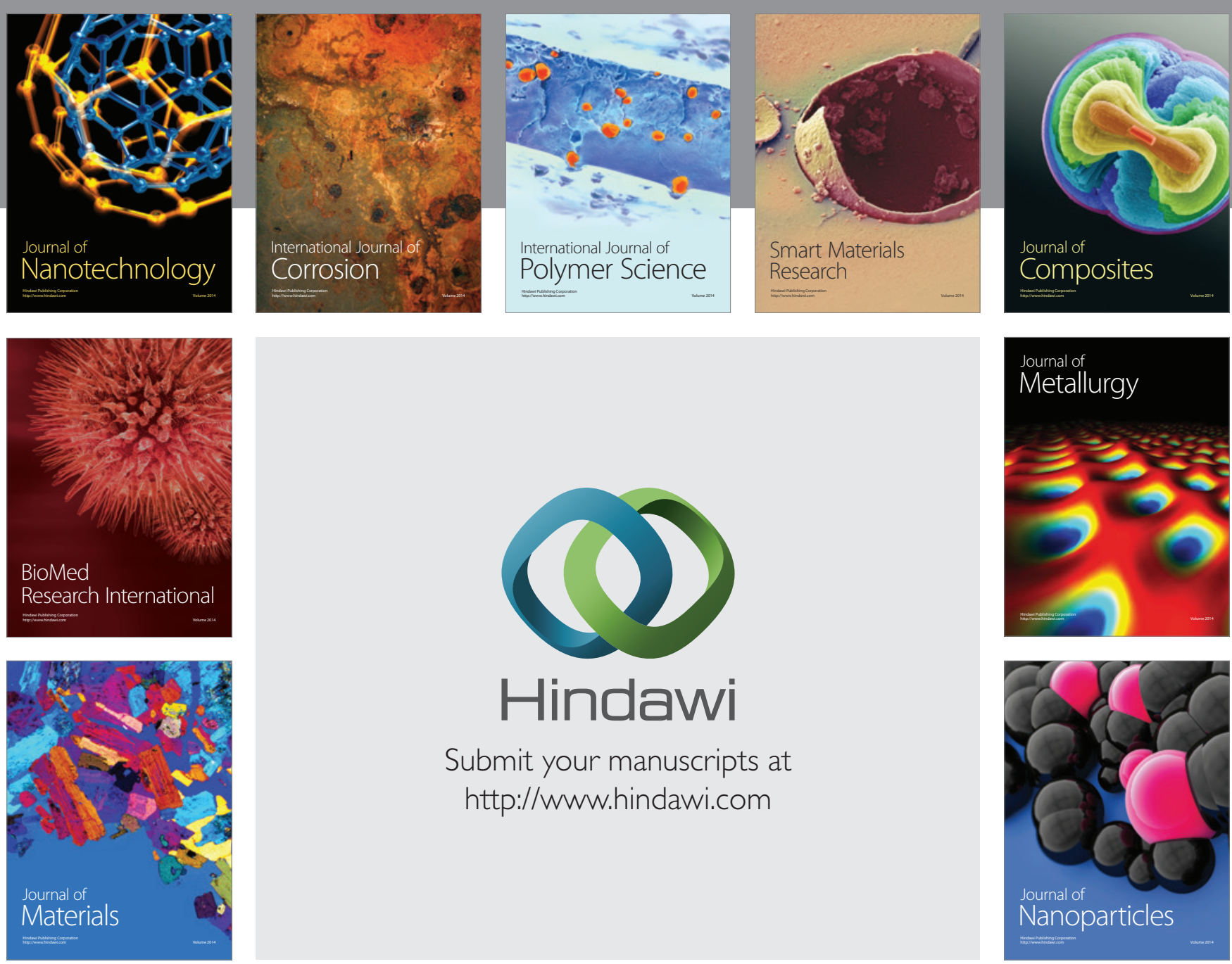

Submit your manuscripts at http://www.hindawi.com
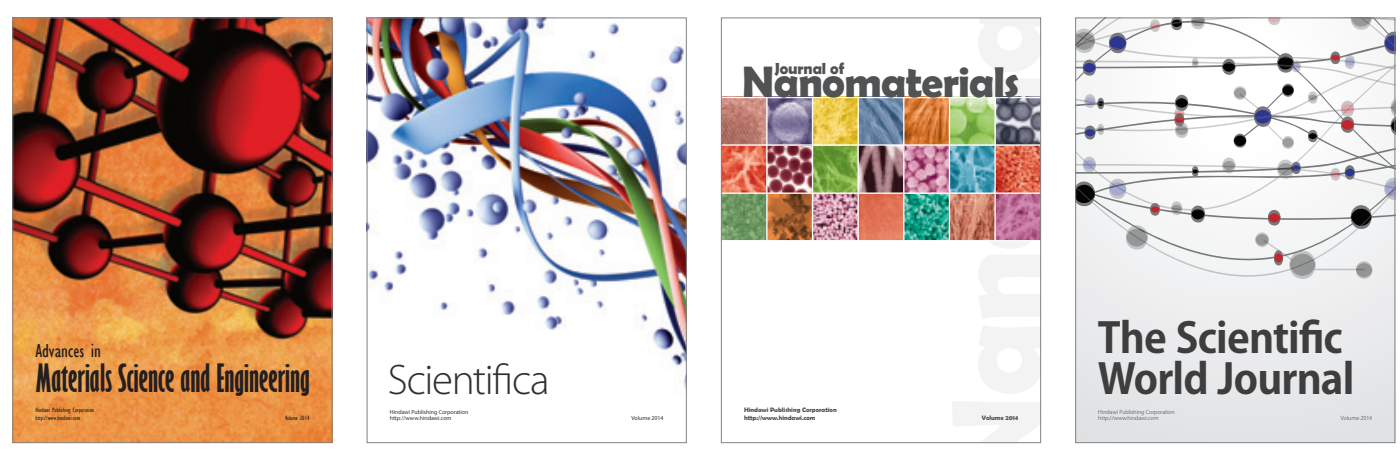

\section{The Scientific World Journal}
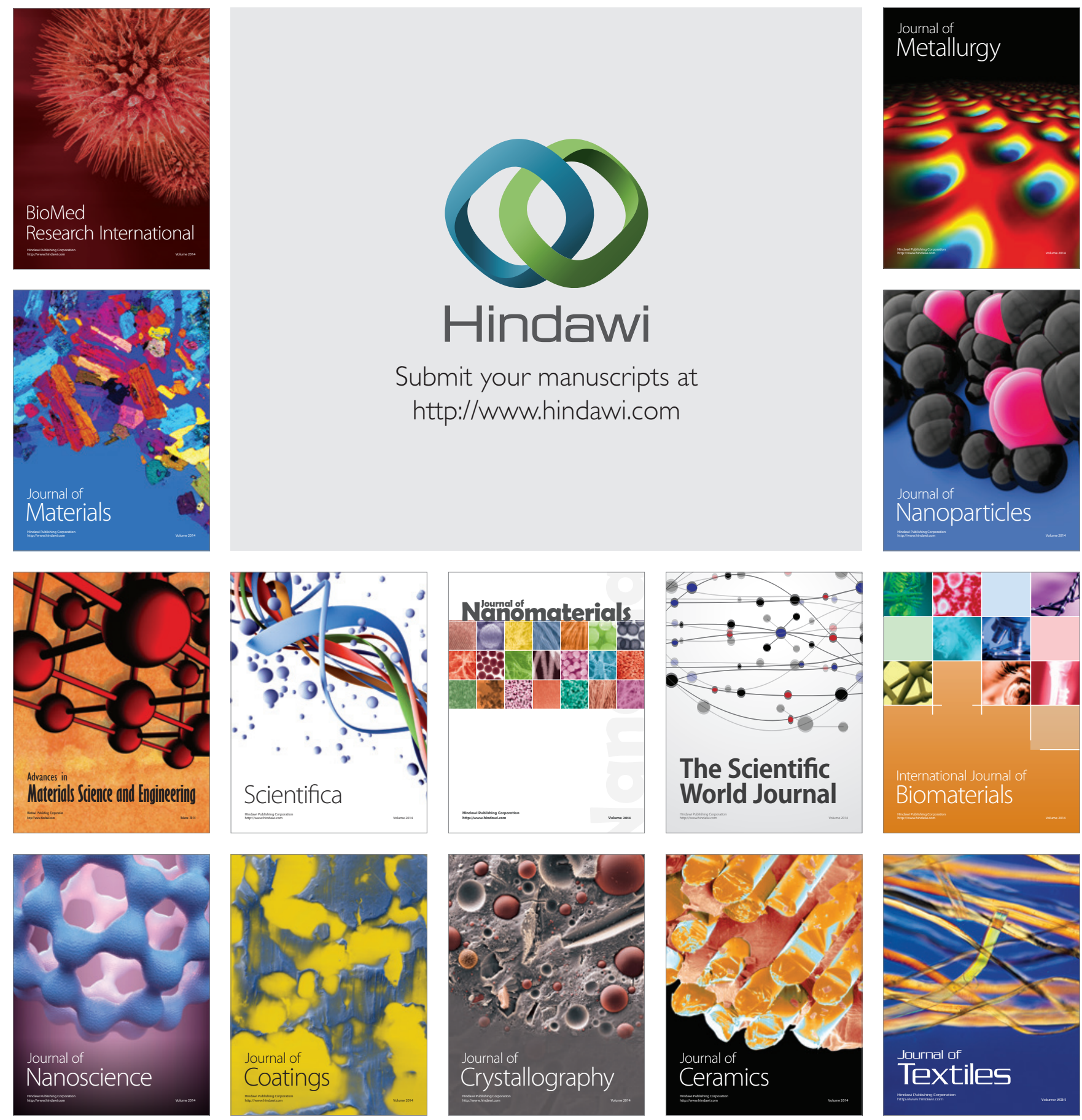